\title{
Inteligencia Emocional: La nueva fórmula del éxito empresarial turístico en Cuba
}

\author{
Emotional Intelligence: the new formula for tourism business success in \\ Cuba
}

\section{Yulima Daimet Valdés Bencomo. ${ }^{1}$, Miguel Ángel Bulit Villafaña ${ }^{2}$, Cinthia Balcárcel} Mara. ${ }^{3}$ \& Luis Efraín Velasteguí López. ${ }^{4}$

\begin{abstract}
The next work presents the relation between the emocional intelligence ante companies, making emphasis on the sector of tourism. It was respected on every moment the historical-logical focus, keeping a cronological order of facts to a better understanding. The main objective of the article is to make known a term that is getting stronger and stronger in the business world, while we drive the current companies and the ones that are about to begin., to give more importance to this term inside the strategies and intern improvement tools. It is precise the base of the emocional intelligence concept, due to the non-exixtence of an only concept at present. It shows the benefits that offer the implementation and control of the emocional intelligence in a company, and also what can happen if this important element is not taken seriously. It can see ways of acting on key situations in any place and even some tools to use by somes managers of determined areas. It concludes about the importance that have this term for the business success of touristics companies, taking as a reference the intern client and the extern client.
\end{abstract}

Key Words: Emocional intelligence, business success, intern client, extern client, results

\footnotetext{
${ }^{1}$ Universidad de la Habana. La Habana, Cuba.yulima40@gmail.com, Orcid: 0000-0003-4477-166x

${ }^{2}$ Universidad de la Habana. La Habana, Cuba. miguelito42@gmail.com, Orcid: 0000-0003-0068-0053

${ }^{3}$ Universidad de la Habana. La Habana, Cuba. cinthiabm1508@ gmail.com, Orcid: 0000-00030191-3250

${ }^{4}$ Ciencia Digital Editorial, Ecuador, luisefrainvelastegui @ cienciadigital.org
} 


\section{Resumen}

El siguiente trabajo presenta la relación que existe entre la inteligencia emocional y las empresas, haciendo énfasis en el sector del turismo. Se respetó en todo momento el enfoque histórico-lógico, manteniendo un orden cronológico de los hechos para mayor comprensión. El principal objetivo del artículo es dar a conocer un término que cobra cada vez más fuerza en el mundo empresarial, mientras impulsamos a las empresas actuales y las que están por comenzar en este mundo, a darle mayor importancia a este vocablo dentro de las estrategias y herramientas de mejora internas. Se precisa la base del concepto de inteligencia emocional, debido a la no existencia de un concepto único en la actualidad. Se muestran los beneficios que brinda la implementación y control de la inteligencia emocional en una empresa, y también lo que puede ocurrir si no se toma un serio este elemento tan importante. Se analizan modos de comportarse en situaciones claves en cualquier instalación e incluso algunas herramientas a utilizar por algunos encargados de áreas determinadas. Se concluye sobre la importancia que tiene este término para el éxito empresarial de las instalaciones turísticas, tomando como referencia al cliente interno y al cliente externo.

Palabras Clave: Inteligencia emocional, éxito empresarial, cliente interno, cliente externo, resultados.

\section{Introducción}

Se puede decir que, desde los primeros pasos de la inteligencia emocional como concepto, fue presentada al mundo como una forma de inteligencia capaz de influir directamente en el éxito durante la vida de cada persona en mayor medida que otro tipo de habilidades como las intelectuales o las cognitivas. Con esto, la capacidad intelectual, de cierta forma, vio como tuvo que dar un paso al costado para que los factores relacionados con las emociones, como la empatía para establecer relaciones óptimas con otras personas y el manejo adecuado de los sentimientos propios y ajenos, tomaran más importancia en la sociedad.

Los primeros estudiosos referentes a la inteligencia emocional enfocaron su trabajo en el desarrollo teórico de modelos y en crear instrumentos que permitan evaluar y medir rigurosa y eficientemente el nivel de inteligencia emocional. En la década de los noventa, este tema alcanzó la cima de la popularidad mundial, en gran parte gracias a Daniel Goleman (1995) y su libro "Inteligencia Emocional: ¿Por qué puede importar más que el concepto de cociente intelectual?", con el cual expandió el concepto y lo dió a conocer tras bastante tiempo en el anonimato para la mayoría.

Esta explosión fue gracias a un relevante artículo de Nancy Gibbs, en 1995, sobre este libro de Goleman y fue el primer medio de comunicación interesado en la inteligencia emocional. Posteriormente, los artículos sobre este tema comenzaron a aparecer cada vez con mayor frecuencia a través de una amplia gama de entidades académicas y puntos de 
venta populares. Es al psicólogo estadounidense Daniel Goleman a quien se le atribuye la popularización del vocablo inteligencia emocional, el citado especialista explica que: "el éxito de una persona no depende tanto de su coeficiente intelectual o de sus estudios académicos, si no de la inteligencia emocional y la capacidad de reconocer los sentimientos propios y los de los demás, para así manejar bien las emociones y tener relaciones más productivas con quienes nos rodea”. (Goleman 2001, p.3).

Desde esos momentos de explosión popular hasta el día de hoy, son muchos los estudios e investigaciones científicas que han intentado unificar criterios y arribar a un concepto único de inteligencia emocional, pero actualmente, tras muchas variaciones y aportes, esto no ha sido posible. Esto se debe, en gran medida, a la cantidad de autores que dedicaron parte de su vida al estudio de este concepto, y al hecho de que el mundo emocional no es una ciencia exacta, no tiene un método de medición absoluto, y cuando las conclusiones sobre un tema se basan exclusivamente en criterios, es obvio que será difícil, quizás imposible, llegar algún día a una unificación. Entre todos estos autores cabe mencionar a Howard Gardner (1985), Peter Salovey (1991), John Mayer (1991) y Daniel Goleman (1995), ya mencionado anteriormente.

La inteligencia emocional ha comenzado a tomar más relevancia en el funcionamiento de las empresas actualmente. Los grandes estudiosos de la misma como concepto, se dieron cuenta de la influencia que tiene sobre el éxito, tanto personal como laboral y todo esto por encima de otras habilidades como las intelectuales y las cognitivas, que durante mucho tiempo eran las más importantes dentro de este sector. El manejo de las emociones, la empatía para relacionarse con el resto de compañeros o la resolución de conflictos internos comenzaron a obtener protagonismo en el modelo empresarial actual, debido a la importancia a nivel social de cada una de estas características.

\section{Desarrollo}

Los estudios respecto a la inteligencia emocional no han quedado solo en cómo pueden afectar a la vida diaria de las personas, sino que, con el paso del tiempo, ha crecido el interés por analizar detalladamente la relación entre la inteligencia emocional y otros términos como la inteligencia empresarial o la inteligencia social.

Este interés por estudiar cómo afecta directamente la inteligencia emocional al éxito empresarial viene dado por investigaciones más recientes como la de Damasio y Evans que demuestra que las emociones son claves en el proceso de toma de decisiones, ya que para Damasio la ausencia de emoción y sentimiento puede romper la racionalidad y hacer casi imposible tomar una decisión sabia. Este tipo de estudios son los que han dado el impulso necesario para que los directores de empresas o incluso los nuevos emprendedores, se tomen la inteligencia emocional con más seriedad cuando se trazan objetivos y confeccionan el proyecto a seguir. 
En todas las empresas es de vital importancia que las personas, desde cuadro directivo hasta personal de contacto, sean capaces de gestionar correctamente habilidades como expresar cada emoción con precisión, conectar con los sentimientos ajenos o regular las emociones propias para propiciar un crecimiento emocional e intelectual. Esta gestión de habilidades permite a cada uno poder analizar correctamente una circunstancia determinada y también aprovechar las oportunidades que se puedan presentar tanto a nivel personal como laboral. Además, el principal objetivo de cada empleado debe ser convertirse en una persona emocionalmente competente, a través de un cambio de actitud ante las dificultades.

Esta influencia en la toma de decisiones es lo que ha hecho que la inteligencia emocional tome más protagonismo en las empresas, debido a que cada vez es más importante para la obtención de resultados y para conseguir la satisfacción plena del cliente, que, al fin y al cabo, es de quien depende el éxito empresarial. Este protagonismo en el modelo empresarial se puede observar de dos maneras: mediante el uso de métodos para evaluar la inteligencia emocional en el reclutamiento de personal y mediante la implementación de técnicas y herramientas para mejorar el nivel de inteligencia emocional de los empleados ya contratados.

Estos mecanismos se ven incluso con mayor fuerza en las empresas turísticas. Es un hecho que el turista actual quiere algo más que solo salir de vacaciones o escaparse de la rutina diaria, y es que busca también emociones para su enriquecimiento personal y para su propia felicidad. Según Barcial (2005), el turista es un consumidor perfectamente diferenciado, que lo que busca es atesorar vivencias únicas e individuales, tanto en lo lúdico como en lo educativo y cultural. Cada cliente quiere encontrar experiencias positivas para rentabilizar al máximo ese tiempo que planificaron con antelación y disfrutarlo en un ambiente acogedor.

El buen manejo de la inteligencia emocional en el personal de contacto es esencial para toda empresa turística, ya que la calidad de servicio que percibe cada cliente es lo que va a definir en mayor medida su nivel de lealtad con respecto a la instalación. Las empresas insisten cada vez más en la importancia de centrarse en las emociones y comportamientos que muestran los empleados, y por tanto del trabajo emocional que ellos mismo realizan, debido a que expresar unos sentimientos adecuados resulta fundamental para lograr que el cliente quede satisfecho y perciba como positiva la experiencia turística.

Todavía no se puede decir que existe solo una manera de obtener el mayor número de clientes posibles en una instalación turística, pero sí es cierto que mediante algunas herramientas se puede mejorar la experiencia del cliente y, por tanto, mejorar la imagen de la empresa, que es lo que progresivamente dará mayores resultados. Como se explica anteriormente, el proceso de reclutamiento es esencial para mejorar el nivel de inteligencia emocional en una instalación, debido a que, mediante los métodos adecuados, puedes integrar al personal de trabajo a nuevos empleados que ya pueden aportar sus habilidades y conocimientos en este aspecto. El departamento de Recursos Humanos de 
cada instalación debe hacer énfasis en la inteligencia emocional durante cada entrevista de trabajo.

Esto es algo que no puede pasar desapercibido, porque las personas emocionalmente inteligentes tienen mayor facilidad para adaptarse a nuevos entornos, a nuevos colegas de trabajo y a todo tipo de clientes. Mientras que, por otra parte, las personas que no posean un nivel de inteligencia emocional adecuado podrían ocasionar conflictos internos a mediano o a largo plazo, debido a sus dificultades para gestionar relaciones y soportar situaciones estresantes.

Los encargados del departamento de Recursos Humanos, específicamente los que tienen que ver directamente con las entrevistas de trabajo deben evaluar de manera rigurosa a la persona entrevistada para el puesto, debido a que muchas personas, conscientes de la importancia de la inteligencia emocional para las empresas, han aprendido a fingir ser emocionalmente inteligentes y a pasar ciertos test con facilidad. En muchas ocasiones logra evadir con éxito ciertos temas que son vitales para obtener un resultado conveniente o incluso han aprendido a dar las respuestas que el evaluador quiere escuchar.

Dentro de la empresa, el manejo adecuado de las emociones es un tema bastante complejo de controlar, por lo que es necesario que la empresa se enfoque en la elaboración de estrategias novedosas y la adquisición de herramientas para lograr el desarrollo de la inteligencia emocional. Para todo aquel encargado de capacitar al personal del sector turístico, debe constituir una prioridad impulsar decisivamente en las empresas, programas que ayuden efectivamente al desarrollo de la inteligencia emocional entre los empleados de las empresas turísticas. Actualmente en Cuba se están llevando a cabo estudios profundos sobre la situación de ciertas empresas respecto a la inteligencia emocional. Esto es de vital importancia en toda instalación porque permite el constante crecimiento del personal interno y mantiene el nivel con respecto a la competencia, que con el paso de los años es cada vez mayor en este aspecto.

Según Carmona, Vargas y Rosas (2015) entre la inteligencia emocional y el desempeño laboral existe una gran relación, es decir cuánto más complejo sea el trabajo, se hace más necesaria la inteligencia emocional y no pueden existir de un modo independiente tanto una como la otra. Ello permite inferir que una adecuada inteligencia emocional es la base para el desarrollo e implementación de comportamientos eficaces, los cuales influyen de manera positiva en los resultados en la organización como el desempeño laboral.

Las personas que deciden desarrollar su inteligencia emocional llegan a mostrar la capacidad para generar las mejores soluciones posibles en situaciones problemáticas o de riesgo, saben trabajar sobre las prioridades, debido a que son conscientes de que todo el trabajo no lo pueden hacer solos. Estos son algunos de los motivos por los que cada organización debe incluir a la inteligencia emocional y a todos los elementos que la componen como una de las competencias a evaluar durante cada proceso de evaluación de desempeño. Mediante esta detallada evaluación podrían identificar a los colaboradores 
que pueden convertirse en líderes de cada equipo de trabajo y tomar decisiones inteligentes para incrementar la productividad y competitividad en las organizaciones.

Mayer, Salovey y Caruso (2004) exponen que después de varios estudios sobre cómo la inteligencia emocional influye en el ámbito laboral, se ha observado que el estado emocional del trabajador no es tenido en cuenta durante la evaluación del desempeño laboral ya que se espera que en este ámbito la persona sea más racional que emocional, y se cree que las emociones pueden afectar negativamente en su desempeño. Las emociones son parte importante del proceso del pensamiento de la persona, y que ignorarlas implicaría dejar de lado la sabiduría de las emociones propias y ajenas e invitar al fracaso como persona y trabajadores de la empresa.

Para toda organización turística el principal objetivo es la satisfacción plena del cliente externo, por tanto, es totalmente necesario que los programas de formación, capacitación y perfeccionamiento de los trabajadores del turismo aborden la temática de la inteligencia emocional, porque el desarrollo y entrenamiento de la misma posibilitaría la prestación de un servicio de excelencia, además de elevar el sentido de pertenencia del cliente interno y fomentar un clima laboral agradable y satisfactorio.

En cada empresa, los empleados son los primeros que deben tener presente este aspecto de la inteligencia emocional y lo que representa para su empresa y la obtención de resultados. Cualquier comportamiento que puedan tener los empleados de la instalación en un momento determinado puede verse afectado por su estado de ánimo e interferir negativamente en la resolución de un conflicto o en una decisión que tienen que tomar en una situación dada.

Para poder ofrecer una buena imagen al cliente externo es clave la capacidad de automotivarse en cada empleado y no trasladar los problemas personales al ámbito laboral, deben ser empáticos y optimistas, además de llevarse bien con cada uno de los clientes atendidos. Hay comportamientos negativos como la apatía, prepotencia, frialdad, impaciencia y excesivo apego a las normas, que provocan el disgusto de cualquier cliente y en consecuencia los alejan y enfurecen, perdiendo la oportunidad de repitencia en la instalación.

Al mostrar optimismo, seguridad y control emocional, los empleados pueden manejar de manera más sencilla y práctica cualquier situación, por difícil que parezca mediante el adecuado desarrollo de sus habilidades sociales, comunicación asertiva y manejo de conflicto. La capacidad para manejar correctamente las habilidades correspondientes a la inteligencia emocional debe estar presente en cada uno de los empleados, para que el cliente se lleve una experiencia total y que los conflictos internos se resuelvan con todos apuntando a la misma dirección y con la suficiente claridad.

Uno de los elementos más importantes a tener en cuenta es este aspecto, es que los conflictos internos no deben afectar la experiencia final del cliente, todos deben tener la 
suficiente habilidad para resolverlos sin afectar el servicio. Además, para el personal de contacto es vital dejar los problemas personales en casa, porque no saber lidiar con el estrés provocado por asuntos externos, afecta enormemente en los resultados empresariales.

Queda claro que la inteligencia emocional es la capacidad para reconocer las emociones propias y ajenas, pero es importante saber qué sucede cuando esto se convierte en un requisito fundamental para llevar el control de una organización. El líder de una empresa que busca el éxito no es aquella persona que solamente da órdenes y en ocasiones incluso pierde el control de la situación sin enseñar nada, estas características se asemejan más a las de un jefe. Según Soriano (2016) el líder es aquel que enseña el camino, el que muestra con ejemplos y se compromete en el logro de los objetivos.

Visto desde la perspectiva empresarial, el liderazgo es una pieza imprescindible si tiene como enfoque contribuir de forma positiva en el colaborador. El concepto de líder y liderazgo son diferentes y de reciente creación por los diversos autores que en su mayoría definen al liderazgo como un conjunto de habilidades que se relacionan con una forma de guiar un grupo, mientras que un líder es la persona capaz de hacer que las demás se apasionen, motiven y se interesen por una actividad o misión a cumplir, sin ser necesario imponer, difundir el miedo y esto se logra con motivación, convencimiento, y persuasión.

Ser capaz de reconocer y gestionar las emociones propias y la de los clientes o compañeros de trabajo, además de tener la capacidad de potenciar esas características es fundamental para la formación de un líder dentro de una organización. Estas características les permitirán a los empleados comunicarse de forma eficiente con su entorno, reconocer las necesidades suyas y de la organización, así como priorizar responsabilidades dentro del ámbito laboral. Los líderes son los que consiguen potenciar mejor todas estas habilidades con el fin de alcanzar el éxito mediante el sobrecumplimiento de los planes institucionales.

Según Hochschild (1983) los empleados que son "inteligentes" con sus emociones, suelen ser más eficientes y eficaces en sus interacciones con el ambiente de trabajo y con sus compañeros de trabajo, ejemplos típicos son el entusiasmo en las personas de ventas, la perseverancia en los cobradores, y la empatía en los trabajadores del sector turístico.

Investigando de manera más profunda en esta relación, diversos trabajos destacan su importancia por encima del efecto que tiene sobre el mismo la inteligencia cotidiana. Así, Van Rooy y Viswesvaran (2003) aseguran que la capacidad cognitiva sólo supone el 25 por ciento de la variación en el desempeño laboral, mientras que Goleman (1998) encuentra que el 67 por ciento de las habilidades consideradas esenciales para cumplir de manera eficaz eran aptitudes emocionales. 
Es por lo antes expuesto que cada vez hay más empresas que durante sus procesos de selección de personal, se enfocan en el desempeño de cada candidato durante situaciones de alta exigencia, estrés o incomodidad, para poder examinar su capacidad para gestionar sus emociones en estos momentos clave en toda organización. Estos nuevos métodos no consisten solamente en mejorar el nivel de inteligencia emocional interna, sino también encontrar potenciales líderes que puedan guiar a las próximas generaciones de empleados y formar una base consolidada y estratégica respecto a la inteligencia emocional en todos los niveles.

Esto quiere decir que las empresas no buscan solamente personas que estén capacitadas intelectual y técnicamente para asumir un puesto de trabajo determinado, sino que también valoran de una manera positiva todas estas características que permitan crecer a la empresa en el aspecto emocional, tanto interna como externamente.

Durante ya más de un año, las condiciones han cambiado aún más para el sector empresarial y con mayor incidencia en el sector turístico debido a la pandemia mundial que ha azotado al ser humano y a la economía global: el Covid-19. El principal problema de esta pandemia fue su llegada de manera inesperada, lo cual provocó un duro golpe a todo el sector empresarial. En Cuba, muchos trabajadores tuvieron que ser reubicados, los ingresos disminuyeron considerablemente, la productividad se vió afectada, llegando a niveles de deterioro total, por lo que las empresas más capaces comenzaron a trazar estrategias para evitar unas caídas más grandes o incluso la disolución. Cada organización debe tener siempre un plan para enfrentar, desde simples emergencias, hasta catástrofes, como esta pandemia.

No solo existieron problemas en las empresas internamente, sino que el turismo decreció considerablemente. Cuba pasó de ser uno de los países que mejor controló la pandemia en el continente y en el mundo a ver como una tercera ola consumió a la isla en una nueva crisis económica. Muchos viajeros dejaban de viajar por temor y las instalaciones turísticas vieron como el flujo turístico comenzó a descender drásticamente. También sucedió que muchos que sí tenían interés en visitar el país, no lo hacían por los necesarios protocolos de salud y protección, incluyendo cuarentenas obligatorias que, obviamente, no llamaban la atención de los clientes.

Estos elementos externos fueron los causantes de gran parte de las pérdidas económicas en las instalaciones turísticas, porque a pesar de los pocos ingresos, deben seguir gastando en mantenimiento o en inversiones ya programadas anteriormente, lo cual provocó un enorme daño financiero en el sector turístico cubano.

Uno de los factores que ha golpeado considerablemente a las empresas turísticas ha sido la restricción general de viajes y las cuarentenas internas, debido a que provocan afectaciones en la mano de obra de la empresa, interrumpiendo las cadenas de suministro justo a tiempo y desencadenando advertencias de ventas en las diferentes industrias. 
Además de las propias restricciones legales, hay muchas personas que se ausentan por miedo o por simplemente cumplir con las medidas orientadas de quedarse en casa, pero una de las peores cosas que puede hacer la empresa en estos casos, es obligar al trabajador a asistir sin cumplir los protocolos correspondientes y atentando a la protección del empleado.

Más allá de las preocupaciones que se tienen regularmente en las empresas sobre la continuidad operativa del negocio, la protección de los empleados y la preservación del mercado, los negocios, y los países, deben analizar nuevamente su exposición a interdependencias complejas y en evolución que podrían agravar los efectos de las pandemias y otras crisis. Toda empresa que considere invertir en resiliencia organizacional van a estar mejor preparadas para combatir la adversidad y recuperarse de los daños ocasionados.

A causa de toda la crisis que ha provocado la pandemia, la resiliencia ha sido uno de los términos más utilizados para referirse a las características de los seres humanos que les permite hacerle frente a un problema y solucionarlo de la mejor manera posible, y también se utiliza para referirse a las organizaciones, las cuales deben aplicar estas características si quieren mantenerse en pie.

Esta característica cuando se enfoca en las empresas se define como resiliencia organizacional según la ASIS Internacional y se refiere a la capacidad de una organización para recuperarse, por sí misma, de cualquier interrupción de sus actividades, internas o externas, comprometiendo a todos sus medios. El nivel de resiliencia de una organización se define por su velocidad y agilidad para retornar a una situación de normalidad, pero también por la capacidad de crear las condiciones necesarias para evitar daños por futuros inconvenientes.

El primer paso para todas las empresas afectadas por la pandemia es conseguir el regreso de sus trabajadores. Para esto, dada la situación, deben asegurar una transición confiable de regreso a los puestos de trabajo con protocolos acordes a la situación de su país en particular y una supervisión adecuada al estado de salud de cada trabajador y su seguridad. Este proceso requiere capacidades nuevas o mejoradas en esta materia, incluidas las pruebas, la certificación, la planificación de la capacidad y los cambios en la distribución del espacio de trabajo para limitar las interacciones entre grupos. Estas estrategias le podrán proporcionar al empleado la confianza necesaria para regresar a su puesto laboral y trabajar en un ambiente más seguro para él y para su familia.

Las empresas deben tener clara la situación provocada por una crisis determinada y manejarla con inteligencia emocional, para así poder salir de situaciones difíciles como la provocada por el Covid-19. Es importante entender las emociones, de donde vienen, y poder controlarlas para finalmente convertirlas en herramientas que ayuden a superar este tipo de momentos. 
Una de las principales moralejas que podemos obtener de esta crisis pandémica tiene que ver con la resiliencia y con la seguridad y protección de activos, y es que no sirve solo una palabra para referir acciones que debieran ser ejecutadas por personas $\mathrm{y}$ organizaciones para volver a la calma pronto, es bastante más. Se trata de pensar, razonar, culturizar, organizar, disponer, planificar, ensayar, testear, evaluar y volver a pensar en un ciclo virtuoso de absolutas dimensiones dinámicas y holísticas.

Existen casos de algunas organizaciones que no hicieron este trabajo previo a la pandemia y muchas terminaron reaccionando e improvisando, incluso algunas han visto como es inevitable su desaparición del sector y del mercado. Esto ocurre, en ocasiones, porque los líderes organizacionales se confían o le restan importancia a la pandemia por tener referencias erróneas o simplemente no tener la cultura necesaria para analizar correctamente la situación.

Esto es un ejemplo claro y actual de que la inteligencia emocional debe estar implementada en la estrategia de cualquier empresa que aspire al éxito, no solo para mantener satisfecho al cliente externo y mantener controlados los conflictos internos, sino que también es vital para minimizar el daño recibido por situaciones inesperadas como la Covid-19 y para recuperar todo lo perdido en el menor tiempo posible.

\section{Conclusiones}

- La inteligencia emocional es un tema que, a pesar de su explosión y crecimiento en el mundo actual, todavía está muy infravalorado por gran parte del sector turístico, lo que provoca que muchas empresas no alcancen los objetivos que se trazaron y no logren tampoco identificar el problema que las está llevando a esa situación.

- Es un elemento vital para contrarrestar situaciones inesperadas y que provoquen daños potenciales a la empresa. Todos los clientes internos de una empresa que aspira al éxito deben navegar hacia la misma dirección para superar cualquier obstáculo externo y poder recuperarse de cualquier daño sufridos.

- Países como Cuba se han visto obligados a implementar estrategias referentes a la inteligencia emocional debido a olas del virus que superaron todos los pronósticos y podían acabar con la economía entera de un país y, por tanto, con las empresas turísticas que no le dieran la importancia necesaria a este aspecto.

Referencia bibliográfica

Alzina, R. B. (s.f.). Psicopedagogía de las Emociones. Sintesis S.A.

Anáhuac, G. (12 de Febrero de 2020). Inteligencia emocional en la empresa. Generación Anáhuac, 2. Obtenido de www.anahuac.mx 
Bertrand. (2016). Psicoligía y Mente.

Concepción, R. (s.f.). La Inteligencia Empresarial: Análisis teórico y pasos para su implementación en el contexto cubano. Revista académica de economía.

Corzo, M. C. (4 de Diciembre de 2014). Gestiópolis. Obtenido de www.gestiopolis.com

Darwin, C. (1872). La Expresión de las Emociones.

Goleman, D. (1995). Inteligencia Emocional: ¿Por qué puede importar más que el concepto de cociente intelectual?

Ifefor. (s.f.). Obtenido de www.ifefor.es

Jacobo, D. J. (2015). Inteligencia Emocional, Rasgos de Personalidad e Inteligencia Psicométrica en Adolescentes. Murcia, España.

John Mayer, P. S. (2004). Emotional intelligence: Theory, findings, and implications.

Patricia Carmona-Fuentes, J. G.-H.-R. (2015). Influencia de la inteligencia emocional en el desempeño laboral. Obtenido de Ecured: www.ecured.cu

Payne, W. (1985). Un estudio de las emociones: El desarrollo de la inteligencia emocional.

Retos Directivos. (15 de Junio de 2015). Obtenido de https://retos-directivos.eae.es

Retos en Supply Chain. (28 de Junio de 2018). Obtenido de www.retos-operacioneslogística.eae.es

Rooy, D. V., \& Viswesvaran, C. (2003). The emotionally intelligent female: a metaanalysis of gender differences.

Sáez, O. J. (2017). LA INTELIGENCIA EMOCIONAL Y SU CONTRIBUCIÓN A LA EDUCACIÓN ARTÍSTICA EN LA UNIVERSIDAD . Revista Conrado.

SeguriLatam. (4 de Agosto de 2020). Obtenido de www.segurlatam.com

Soriano, M. A. (2016). La inteligencia emocional para el liderazgo. Xalapa Veracruz: Universidad Veracruzana.

Valenica, G. F., \& Salazar, J. I. (2012). La Inteligencia Emocional en el éxito empresarial. Quito, Ecuador.

Wikipedia. (s.f.). Obtenido de www.wikipedia.com

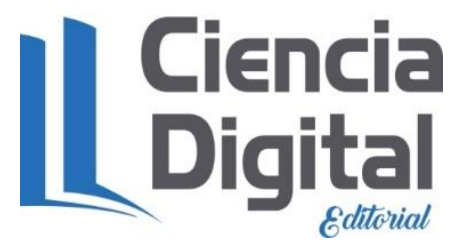




\section{PARA CITAR EL ARTÍCULO INDEXADO.}

Valdés Bencomo, Y. D., Bulit Villafaña , M. Ángel, Balcárcel Mara, C., \& Velasteguí López, L. E. (2021). Inteligencia Emocional: La nueva fórmula del éxito empresarial turístico en Cuba. Explorador Digital, 5(2), 135-146. https://doi.org/10.33262/exploradordigital.v5i2.1692

\section{\Cigncia}

El artículo que se publica es de exclusiva responsabilidad de los autores y no necesariamente reflejan el pensamiento de la Revista Explorador Digital.

El artículo queda en propiedad de la revista y, por tanto, su publicación parcial y/o total en otro medio tiene que ser autorizado por el director de la Revista Explorador Digital.
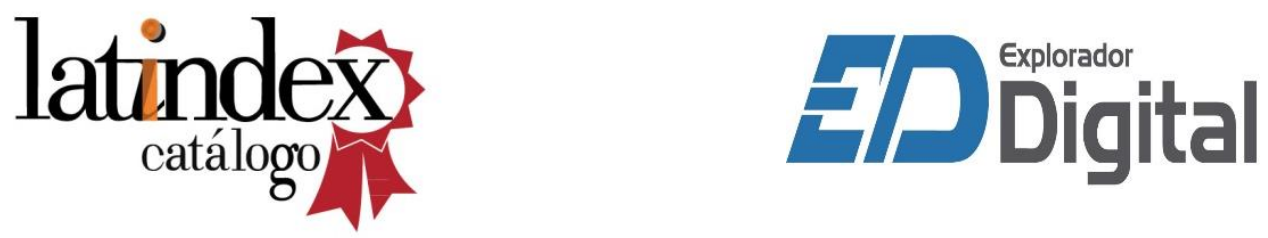